\title{
Review Article \\ Compression of Extreme-Ultraviolet Ultrashort Pulses by Grating Configurations
}

\author{
Fabio Frassetto, Paolo Miotti, and Luca Poletto \\ Institute of Photonics and Nanotechnologies, National Research Council of Italy, Via Trasea 7, 35131 Padua, Italy \\ Correspondence should be addressed to Luca Poletto; poletto@dei.unipd.it
}

Received 17 November 2014; Accepted 7 January 2015

Academic Editor: Khalique Ahmed

Copyright ( 2015 Fabio Frassetto et al. This is an open access article distributed under the Creative Commons Attribution License, which permits unrestricted use, distribution, and reproduction in any medium, provided the original work is properly cited.

\begin{abstract}
The design and realization of grating instruments to condition the spectral phase of ultrashort extreme-ultraviolet pulses are discussed. The main application of such configurations is the temporal compression of pulses by compensating the phase chirp and getting close to the Fourier limit. We discuss the two configurations useful for the realization of ultrafast grating compressors, namely, the classical diffraction mount and the off-plane one. The configuration may be applied to free-electron lasers and highorder laser harmonics.
\end{abstract}

\section{Introduction}

The developments in laser technology over the last years lead to the generation of extreme-ultraviolet (XUV) and X-ray coherent ultrashort pulses in the femtosecond and subfemtosecond time scale $\left(1 \mathrm{fs}=10^{-15} \mathrm{~s}\right)[1,2]$. While femtosecond optical lasers have offered unique insights into ultrafast dynamics, short wavelength radiation offers the capability to access and measure the structural arrangement and electronic structure; therefore, the advent of high-energy, short-pulse Xray sources based on free-electron lasers (FELs), high-order laser harmonics (HHs), and laser plasmas is now making it possible to probe the dynamics of electrons within molecules $[3,4]$. In the following, we will focus our attention on HHs generated in gas and FELs, as ultrashort sources with high brightness, high degree of coherence, and high peak intensity.

The $\mathrm{HH}$ spectrum is described as a sequence of peaks corresponding to the odd harmonics of the fundamental laser wavelength and having an intensity distribution characterized by a vast plateau, whose extension is related to the pulse intensity and frequency. The use of advanced phase matching mechanisms and interaction geometries has made possible the generation of $\mathrm{HHs}$ up to the water window region $(2.3-4.4 \mathrm{~nm})$, while still using a table-top laser source [57]. The radiation generated with the scheme of the HHs using few-optical-cycles laser pulses is currently the main tool for the investigation of matter with attosecond resolution ( 1 as $\left.=10^{-18} \mathrm{~s}\right)[8-11]$. Both trains $[12,13]$ as well as isolated [14-18] bursts of attosecond pulses have been experimentally demonstrated. Recently, circularly polarized attosecond pulses have been also demonstrated $[19,20]$. The physical grounds of such short pulses originate from the model of HHs generation, that is, the phase-matched emission of radiation as result of the recombination of a tunnel-ionized electron with its parent ion. Once the conditions for such recombination are realized in only one occurrence per laser pulse, an isolated ultrashort XUV pulse is generated. Both trains and isolated attosecond pulses are positively chirped, resulting from the different duration of the quantum paths that contribute to different portion of the emitted spectrum. A detailed discussion of the attosecond chirp is found in [21]. Due to the chirp, the pulse temporal duration is longer than the Fourier limit. The condition of being at the Fourier limit is equivalent to the condition of a frequency-independent spectral phase and implies that the time-bandwidth product is at its minimum and that there is no chirp. Positively chirped pulses may be temporally compressed down to the Fourier limit by introducing a system that gives a negative chirp that compensates for the intrinsic chirp of the pulse. This problem has been successfully addressed by exploiting the negative group delay dispersion (GDD) of a thin metallic filter (see $[13,15]$ ) or broadband multilayer optics (see [16]). 
The introduction of a compressor tunable at different energies may add strong flexibility to the beamline when different spectral regions of operation are requested.

FEL sources facilities generate spatially coherent XUV/Xray radiation with characteristics similar to the light from optical lasers, ultrashort time duration, and an increase of 6-8 orders of magnitude on the peak brilliance with respect to $3 \mathrm{rd}$ generation synchrotrons, opening the way to new studies in ultrafast phenomena in different fields spanning from biology to physics of matter [22-25]. Several methodologies have been proposed for the generation of ultrashort FEL pulses in the femtosecond and subfemtosecond regime, as time slicing [26-28] or the reduction of the electron bunch charge [29], which has brought to the generation of FEL pulses as short as $3 \mathrm{fs}$ [30]. Most of these methods rely on the selection of an opportunely small portion of the electron beam which undergoes FEL amplification. One of the main drawbacks common to these solutions is the reduction of the amount of charge effectively contributing to the light amplification. An alternative possibility is the optical compression of the radiation pulse generated by the whole electron beam. In this case, the electron beam is required to have a nonzero energy chirp in order to generate a chirped pulse. The FEL pulse has then to be compressed to reach the Fourier limit. As for solid-state lasers, where frequency chirping is introduced to stretch the pulse before its amplification and, then, after amplification, compensated to recover the ultrashort duration and high peak power, chirped pulse amplification may be applied also to FELs, if the seeding pulse is stretched in time before interacting with the electron beam [31-33]. Despite the necessity of an additional optical stage, namely, the FEL compressor, this solution allows the use of the whole electron beam charge obtaining a significantly higher number of photons with ultrashort duration.

Indeed, for both HHs and FEL facilities, the availability of a XUV and X-ray tunable optical compressor is particularly attractive in order to increase the peak intensity and the time resolution of the source.

Here, we discuss the use of gratings at grazing incidence to realize a broadband and tunable XUV compressor for chirped pulses. In the design of instruments for photon handling in the XUV ultrafast domain, some differences with respect to traditional schemes are notable [34, 35]. The first is that, to exploit the very short duration of the pulse, the study of the optical length of the rays gathered by the pupil and their equalization is mandatory. Moreover, the much extended bandwidth available from the coherent XUV sources may be exploited only if the instrument has a rather flat spectral response. Finally, an overall high throughput of the instrument is often a crucial feature to maintain high peak intensity.

The use of gratings at grazing incidence to realize tunable monochromators for XUV ultrafast pulses is well established for both HHs and FELs. When using a grating for ultrafast pulses, the main problem to be faced is the pulse-front tilt that is introduced by diffraction. Two are the options in designing an ultrafast monochromator, namely, the single-grating [36] or the double-grating design $[37,38]$. In the first case, being adopted as single grating, a residual pulse-front tilt has to be accepted at the output of the monochromator. In fact, each ray that is diffracted by two adjacent grooves is delayed by $m \lambda / c$, where $m$ is the diffraction order, $\lambda$ is the wavelength, and $c$ is the speed of light in vacuum. The pulse-front tilt is given by the total difference in the optical paths of the diffracted beam; that is, $\Delta \tau_{G}=m \lambda N / c$, where $N$ is the number of the illuminated grooves. The aim of the design is to find a suitable grating geometry that minimizes the temporal broadening. Single-grating monochromators with response in the range of few tens of femtoseconds are presently used in $\mathrm{HH}$ beamlines [39-41] and FEL facilities [42]. In the second case, the design consists of a pair of gratings to compensate for the pulse-front tilt. The first grating is demanded to perform the spectral selection on an intermediate slit; the second grating compensates for the pulse-front tilt of the diffracted beam by equalizing the length of the optical paths, giving a temporal resolution much higher than the single-grating design. Double-grating instruments have been demonstrated to be very effective for $\mathrm{HHs}$, with time resolution well below 10 fs [43-47]. The choice between the two options has to be performed as a trade-off between efficiency and throughput, which are maximized in the single-grating design, and temporal resolution, which is maximized in the doublegrating design. Double-grating configurations, which are able to preserve the ultrafast duration of the pulse, have been also proposed as beam splitters for ultrafast intense pulses [48] and as IR-XUV beam separators for HHs [49].

In this paper, we report on the use of gratings to realize XUV compressors for the conditioning of the spectral phase of chirped pulses. A brief introduction to the grating geometries that are required to realize XUV ultrafast instruments is given. The concept is applied to the realization of XUV compressors with applications to HHs and FELs. The concept may find suitable applications to ultrafast spectroscopy experiments.

\section{Grating Geometries for Ultrashort Pulses}

Grazing-incidence gratings may be used in two different geometries: the classical-diffraction mount (CDM) and the off-plane mount (OPM) [50].

In the case of the CDM, the geometry is defined on a plane as shown in Figure 1(a). Let us define the incidence angle $\alpha$ and the diffraction angle $\beta$, respectively, as the angles between the direction of the input beam and the normal beam to the grating and the direction of the diffracted beam and the normal beam to the grating, both taken with positive signs. The grating equation for the CDM is $\sin \alpha-\sin \beta=m \lambda \sigma_{\mathrm{CD}}$, where $\lambda$ is the wavelength, $m$ the diffracted order, and $\sigma_{\mathrm{CD}}$ the groove density.

The geometry of the OPM is shown in Figure 1(b). In this case, the incident and diffracted wave vectors are almost parallel to the grating grooves [51]. The direction of the incoming rays is described by two parameters: the altitude and the azimuth. The altitude $\gamma$ is defined as the angle between the direction of the incoming rays and the direction of the grooves. The azimuth $\mu$ of the incoming rays is defined to be zero if they lie in the plane perpendicular to the grating 


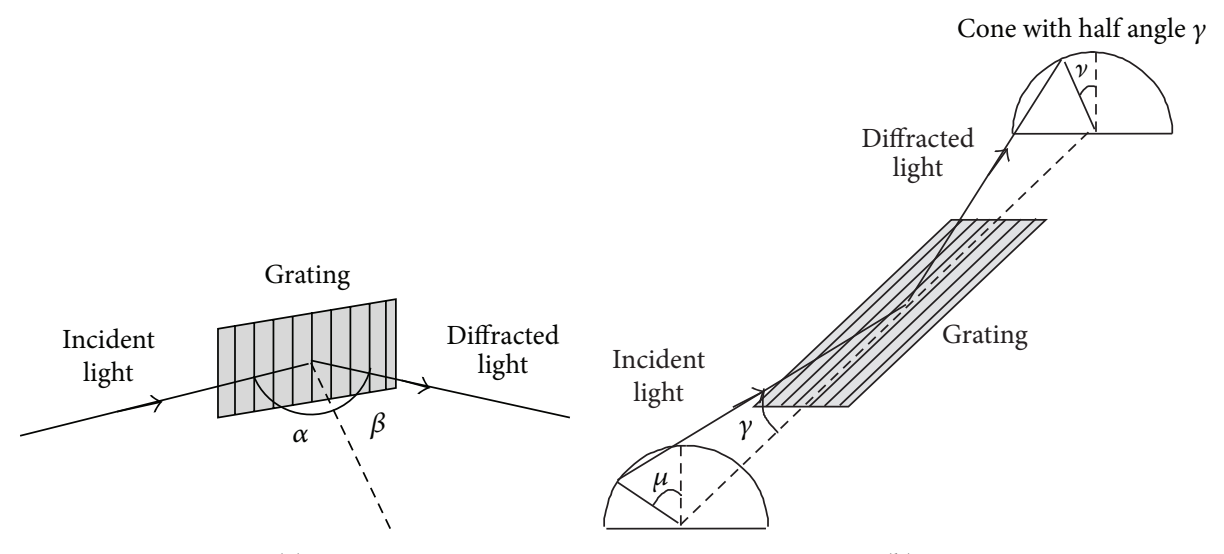

(a)

(b)

FIGURE 1: Reflection grating geometries: (a) classical-diffraction and (b) off-plane mount.

surface and parallel to the rulings. Let $\nu$ define the azimuth of the diffracted light at wavelength $\lambda$ and order $m$. The grating equation for the OPM is $\sin \gamma(\sin \mu+\sin \nu)=m \lambda \sigma_{\mathrm{OP}}$, where $\sigma_{\mathrm{OP}}$ is the groove density. The OPM gives higher throughput than the classical mount, since it has been theoretically demonstrated and experimentally measured that the peak diffraction efficiency is close to the reflectivity of the coating at the altitude angle [52]. Therefore, the OPM geometry is suitable for the design of XUV grating instruments with high efficiency [53].

When realizing a compressor for ultrafast pulses, then the main problem to be dealt with is the pulse-front tilt given by a single grating. This effect, although negligible for picosecond or longer pulses, is dramatic in the femtosecond or even subfemtosecond time scale and has to be corrected by a second grating in a compensated configuration. From the point of view of the ray paths, the differences in the path lengths of rays with the same wavelength within the beam aperture that are caused by the first grating have to be compensated by the second grating; that is, all the rays with the same wavelength make the same optical path. The second grating compensates also for the spectral dispersion caused by the first grating; that is, all the rays at different wavelengths within the pulse spectrum exit the second grating with parallel directions. Both of these conditions are satisfied by a scheme with two equal plane gratings mounted with opposite diffraction orders; that is, the incidence angle on the second grating is equal to the diffraction angle of the first grating. The phase chirp introduced by the system may be finally calculated as the difference in the optical path between rays at different wavelengths. This principle is used in many ultrafast devices in the visible and near infrared, as it was demonstrated that grating pairs may be arranged to realize compensators for the laser cavity dispersion, phase modulators, stretchers, and compressors for chirped pulse amplification [54-56].

\section{Grazing-Incidence Grating Compressor}

The basic version of the arrangement of a XUV grazingincidence grating compressor consists of two identical plane gratings mounted in the compensated configuration, as shown in Figure 2. Since the plane gratings have to be operated in parallel light, a XUV collimating mirror, that is, a paraboloidal or toroidal mirror, is required in front of the compressor. Therefore, the compressor consists of three optical elements, namely, the collimator and the two gratings. Due to the symmetry of the configuration, the output rays are parallel to the input rays for all the wavelengths. Indeed, in the case of the CDM, the incidence angle on G2, $\alpha_{2}(\lambda)$, is equal to the diffraction angle from G1, $\beta_{1}(\lambda)$, and the diffraction angle from G2, $\beta_{2}$, is constant with the wavelength and equal to the incidence angle on G1, $\beta_{2}=\alpha_{1}$. Similarly, in the case of the OPM, the azimuth angle of incident rays on G2, $\mu_{2}(\lambda)$, is equal to the azimuth angle of diffracted rays from G1, $\nu_{1}(\lambda)$, and the azimuth angle of the rays diffracted from G2, $v_{2}$, is equal to the azimuth angle of the rays incident on G1, $\nu_{2}=\mu_{1}$.

Since different wavelengths are diffracted by G1 at different angles, the rays do not make the same optical paths within the compressor. Therefore, the group delay (GD), intended here as the propagation time of the rays from the input plane of G1 to the output plane of G2, is not constant with the wavelength. Aim of the compressor is to introduce suitable group-delay dispersion (GDD), which is a variation of the propagation time of the rays with different wavelengths.

As usual, GD is expressed as a function of $\omega$ :

$$
\mathrm{GD}(\omega) \equiv \frac{\partial \phi}{\partial \omega}=\frac{\mathrm{OP}(\omega)}{c},
$$

where $\mathrm{OP}(\omega)$ is the optical path at frequency $\omega=2 \pi c / \lambda$ and $c$ is the speed of light in vacuum.

Let us define the central pulse frequency $\omega_{c}$ and the central wavelength $\lambda_{c}$ as

$$
\omega_{c} \equiv \frac{\omega_{\min }+\omega_{\max }}{2}=\pi c\left(\frac{1}{\lambda_{\min }}+\frac{1}{\lambda_{\max }}\right), \quad \lambda_{c} \equiv \frac{2 \pi c}{\omega_{c}},
$$

where the bandwidth of the pulse is limited between $\omega_{\min }=$ $2 \pi c / \lambda_{\max }$ and $\omega_{\max }=2 \pi c / \lambda_{\min }$. 


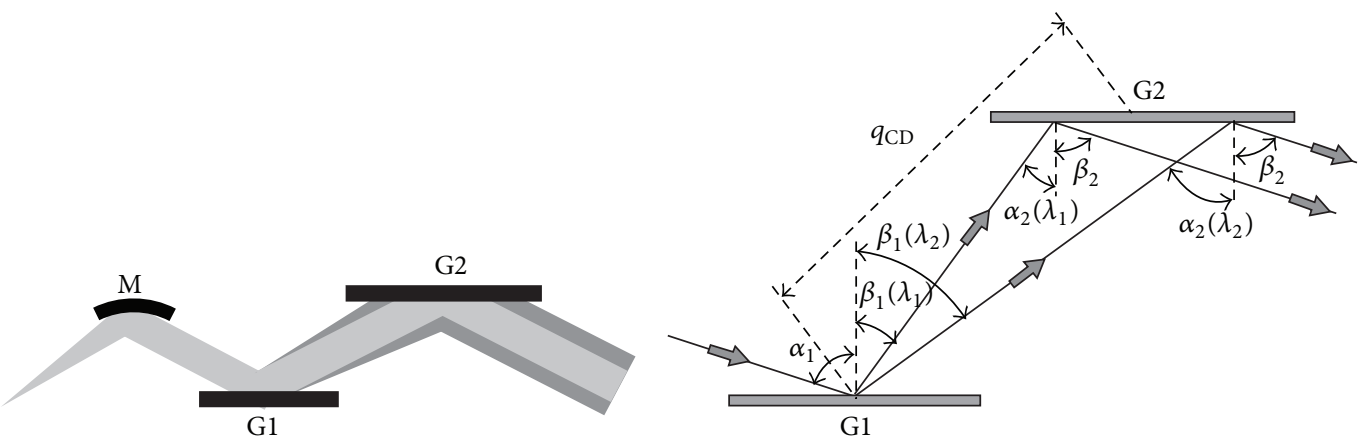

(a)

(b)

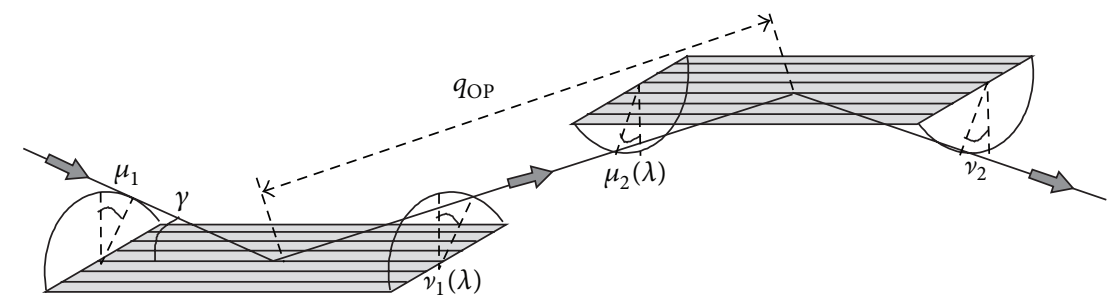

(c)

FIGURE 2: Plane double-grating compressor: (a) schematic view, including the collimating mirror (M), and the two gratings (G1 and G2); (b) classical-diffraction mount; (c) off-plane mount.

In the case of the CDM, the optical path is analytically expressed (for less than a constant term) as

$$
\mathrm{OP}_{\mathrm{CD}}(\lambda)=q_{\mathrm{CD}} \frac{\cos \beta_{c}}{\cos \beta}(1-\sin \alpha \sin \beta),
$$

where $\alpha$ is the incidence angle on G1, $\beta$ and $\beta_{c}$ are, respectively, the diffraction angles from G1 at the generic wavelength $\lambda$ and, at the wavelength $\lambda_{c}$, and $q_{\mathrm{CD}}$ is the G1-G2 distance. In case of a narrow-band pulse with $\Delta \lambda / \lambda<20 \%$, (3) results in linear $\lambda$. Expressing $\beta=\beta_{c}+\Delta \beta$ and using the relation $\Delta \beta=-m \sigma_{\mathrm{CD}} \Delta \lambda / \cos \beta_{c}$, it may be expressed as

$$
\mathrm{OP}_{\mathrm{CD}}(\lambda)=q_{\mathrm{CD}} \lambda_{c}\left(\frac{m \sigma_{\mathrm{CD}}}{\cos \beta_{c}}\right)^{2}\left(\lambda-\lambda_{c}\right) .
$$

Similarly, in the case of the OPM, the optical path is analytically expressed (for less than a constant term) as

$$
\mathrm{OP}_{\mathrm{OP}}(\lambda)=q_{\mathrm{OP}} \frac{\sin ^{2} \gamma \cos \mu}{\cos \nu}(1+\sin \mu \sin \nu),
$$

where the grating parameters have been calculated to have the wavelength $\lambda_{c}$ diffracted at $\nu_{c}=\mu$; that is, $2 \sin \gamma \sin \mu=$ $m \lambda_{c} \sigma_{\mathrm{OP}}$. Expressing $\nu=\nu_{c}+\Delta v$ and using the relation $\Delta \nu=m \sigma_{\mathrm{OP}} \Delta \lambda /(\cos \mu \sin \gamma),(5)$ is linearized in $\lambda$, in case of a narrow-band pulse, as

$$
\mathrm{OP}_{\mathrm{OP}}(\lambda)=q_{\mathrm{OP}} \lambda_{c}\left(\frac{m \sigma_{\mathrm{OP}}}{\cos \mu}\right)^{2}\left(\lambda-\lambda_{c}\right) .
$$

Note that, in both cases, the optical path is anyway increasing with the wavelength and this forces the GDD introduced by the compressor to be negative.
Let us define $K_{\omega}$ as

$$
\begin{aligned}
& K_{\omega}(\mathrm{CD}) \equiv q_{\mathrm{CD}} c\left(\frac{2 \pi m \sigma_{\mathrm{CD}}}{\omega_{c} \cos \beta_{c}}\right)^{2}, \\
& K_{\omega}(\mathrm{OP}) \equiv q_{\mathrm{OP}} c\left(\frac{2 \pi m \sigma_{\mathrm{OP}}}{\omega_{c} \cos \mu}\right)^{2},
\end{aligned}
$$

where $K_{\omega}(\mathrm{CD})$ and $K_{\omega}(\mathrm{OP})$ refer, respectively, to the CDM and to the OPM.

The optical path can be expressed as a function of $\omega$ with the relation

$$
\mathrm{OP}(\omega)=-c K_{\omega}\left(1-\frac{\omega_{c}}{\omega}\right) .
$$

Equation (8) is expanded in series of $\omega-\omega_{c}$ and is integrated to obtain the expression of the phase as

$$
\varphi(\omega)=\Phi_{0}+\Phi_{1}\left(\omega-\omega_{c}\right)+\Phi_{2}\left(\omega-\omega_{c}\right)^{2}+O\left(\omega-\omega_{c}\right)^{4},
$$

where the first coefficient that describes the distortion of the pulse temporal profile is

$$
\Phi_{2}=-\frac{K_{\omega}}{2 \omega_{c}} .
$$

For a narrow-band pulse, the GD is almost linear also in $\omega$ and the GDD is constant and negative; GDD $=-K_{\omega} / \omega_{c}$. Once the required GDD to compress the pulse has been defined, the above equations define the parameters of the grating compressor in both geometries.

Note that, in the CDM, the first grating is normally operated in the order $m=-1$; that is, $\alpha<\beta$, since this gives 


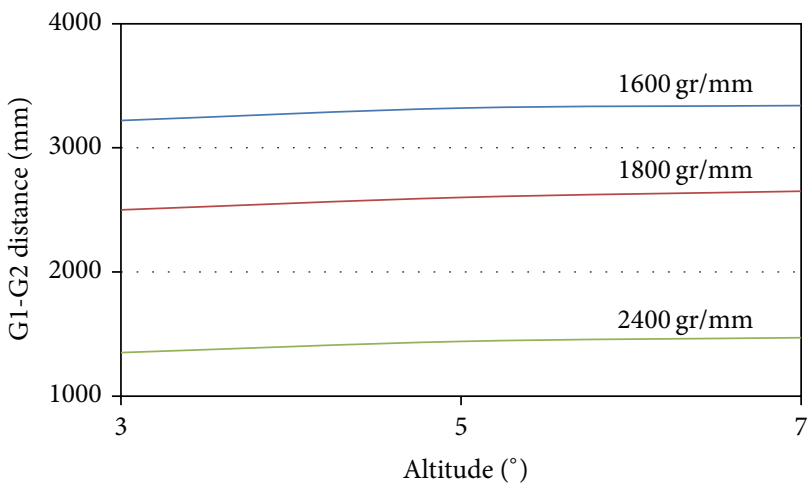

(a)

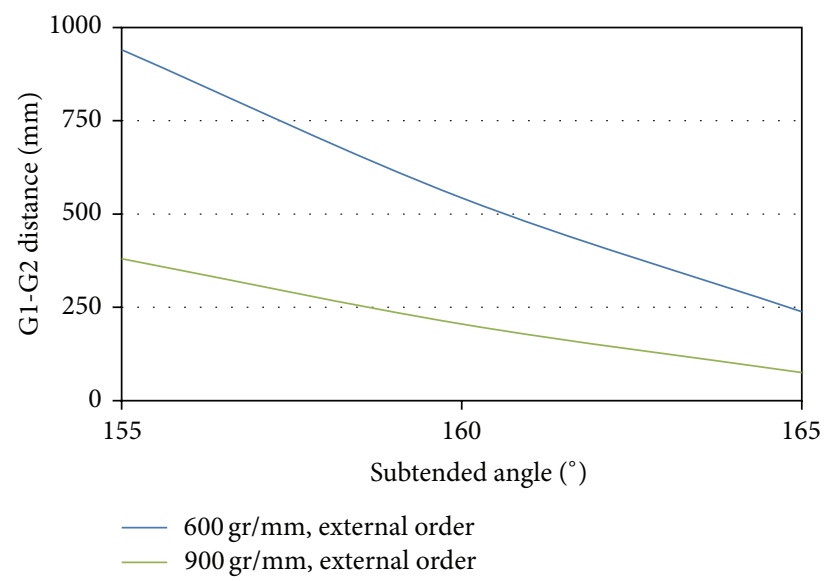

(b)

FIgURE 3: Parameters of the FEL grating compressor: (a) OPM and (b) CDM.

a shorter distance $q$, once the required GDD has been fixed, therefore, a more compact compressor. In the case of the OPM, the operations at order $m= \pm 1$ are totally equivalent.

Once the length of the $\operatorname{arm} q$ has been selected, the two geometries give the same compression if $K_{\omega}(\mathrm{OP})=K_{\omega}(\mathrm{CD})$; that is, $\sigma_{\mathrm{OP}} / \cos \mu=\sigma_{\mathrm{CD}} / \cos \beta_{c}$. Since $\mu$ is typically below $20^{\circ}$ and $\beta_{c}$ is above $80^{\circ}$, the groove density that would be required in the OPM is much higher than the CDM and may not be available from grating providers. Therefore, a compressor in the OPM is typically longer than the corresponding CDM as will be shown in the test case presented below.

The compressor introduces a spatial chirp of the pulse; that is, rays with different wavelengths have the same output direction but they are not exactly superimposed. In the conventional design of compressors for IR pulses, the spatial chirp is canceled by making the beam pass two additional times through the same gratings, so the output spatial dispersion is zero. This cannot be realized in grazing incidence, since it would require the insertion of two additional gratings that would make the configuration too complex. The chirp $\operatorname{SC}(\lambda)$ is expressed, in case of a narrow-band pulse of bandwidth $\Delta \lambda$, as

$$
\begin{gathered}
\mathrm{SC}_{\mathrm{CD}}(\lambda)=q_{\mathrm{CD}} \sigma_{\mathrm{CD}} \frac{\cos \alpha}{\cos ^{2} \beta_{c}} \Delta \lambda \\
\operatorname{SC}_{\mathrm{OP}}(\lambda)=q_{\mathrm{OP}} \sigma_{\mathrm{OP}} \frac{\sqrt{1+3 \cos ^{2} \gamma \sin ^{2} \mu}}{\cos ^{2} \mu} \Delta \lambda,
\end{gathered}
$$

where $\mathrm{SC}_{\mathrm{CD}}$ and $\mathrm{SC}_{\mathrm{OP}}$ refer, respectively, to the $\mathrm{CDM}$ and to the OPM. Since the rays are parallel, the spatial chirp does not influence the quality of the final spot size if the beam is focused by a proper concave mirror.

In the following, we will apply the double-grating configuration to the case of compression of FEL pulses and of attosecond pulses generated through $\mathrm{HHs}$.
TABLE 1: Requirements for the FEL compressor.

\begin{tabular}{lc}
\hline Central wavelength, $\lambda_{c}$ & $13.5 \mathrm{~nm}$ \\
Bandwidth, $\Delta \lambda$ & $0.8 \mathrm{~nm}$ \\
Time stretching, $\Delta t$ & $310 \mathrm{fs}$ \\
Group delay dispersion, GDD & $-37 \mathrm{fs}^{2}$ \\
\hline
\end{tabular}

\section{Grating Compressor Applied to FEL Pulses}

The double-grating compressor, in which optical elements are used at grazing incidence, is very suitable for FEL pulses, since it has robust optical elements to be operated under the intense FEL radiation. Here, we assume the parameters of [57], which are reported in Table 1 and may be a test case for the application to FEL chirped pulse amplification.

The two geometries are compared using commercially available gratings with groove density as high as $2400 \mathrm{gr} / \mathrm{mm}$. The resulting parameters of the compressor are shown in Figure 3. Note that the size of the OPM compressor is much larger than the CDM one. Indeed, the length of the instrument in the CDM may be easily reduced well below $0.5 \mathrm{~m}$ by using a low-density grating; on the contrary, the OPM requires at least about $1.5 \mathrm{~m}$, unless extremely highdensity gratings, which are difficult to be manufactured, are adopted. The spatial chirp is shown in Figure 4. In the OPM case, for the G1-G2 distance being much longer, the spatial chirp is larger. The main advantage of the OPM is the higher total efficiency, which can be quantified in the $0.15-0.20$ range compared to the 0.04-0.07 range of the CDM (see [53]).

It may be concluded that the XUV double-grating compressor for application to FEL radiation on a narrow-band emission requires, in case of the OPM, a definitely larger space than the CDM and gives also a larger spatial chirp. On the other side, the OPM gives higher efficiency. The choice between the two geometries depends mainly on the surrounding conditions, such as the available space on the beamline and the mechanical stability of the optical 


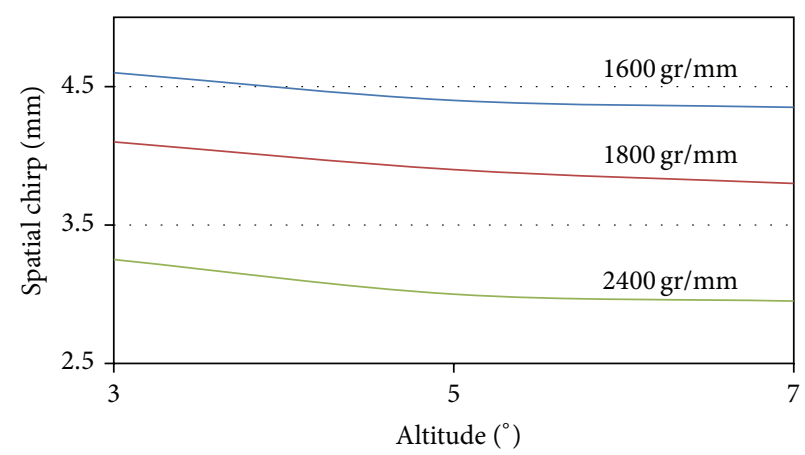

(a)

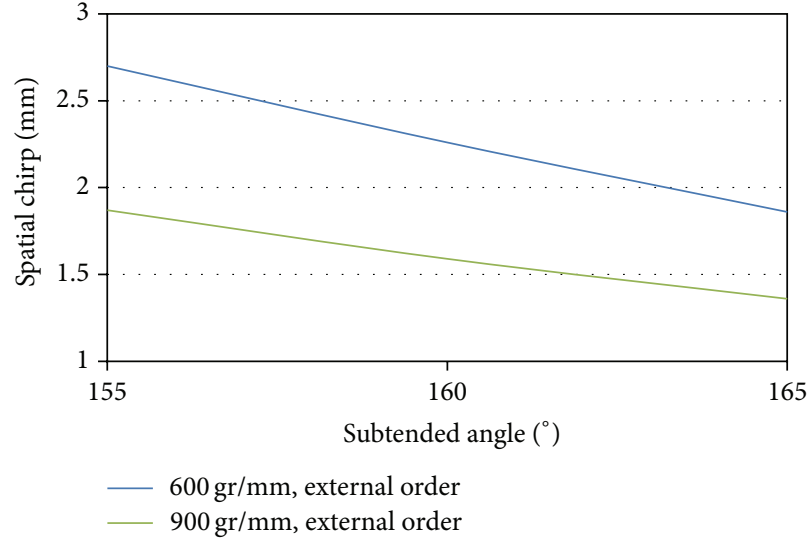

(b)

FIGURE 4: Spatial chirp of the FEL grating compressor: (a) OPM; (b) CDM.

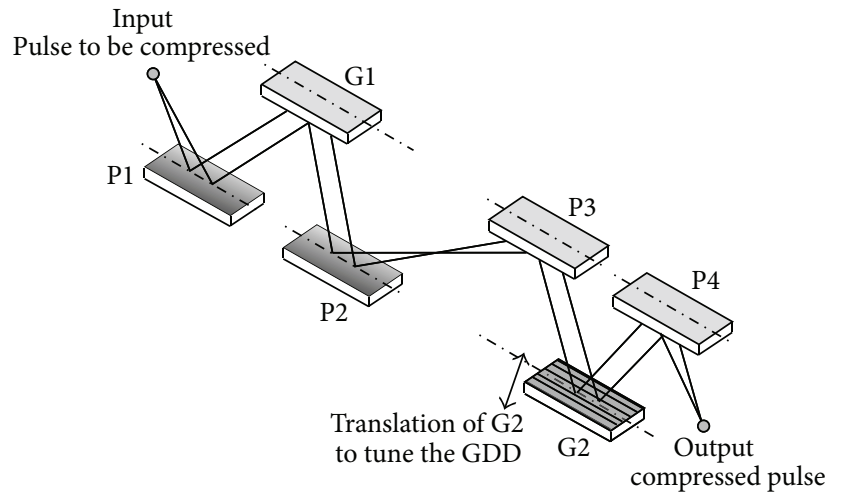

$\mathrm{S} 1=\mathrm{P} 1$ to $\mathrm{G} 1$ distance

$\mathrm{S} 2=\mathrm{G} 1$ to $\mathrm{P} 2$ distance

$\mathrm{S} 3=\mathrm{P} 3$ to $\mathrm{G} 2$ distance

$\mathrm{S} 4=\mathrm{G} 2$ to $\mathrm{P} 4$ distance

FIgURE 5: Layout of the XUV attosecond compressor with gratings in the OPM.

mountings, which may force the choice of the more compact CDM configuration with respect to the OPM.

\section{XUV Attosecond Compressor}

The double-grating configuration shown in Figure 2 is not suitable for the compression of XUV attosecond pulses, since the distance $q$ that is required to give the necessary GDD that compensates for the intrinsic pulse chirp is too small to be realized in practice. Therefore, the configuration has to be modified as shown in Figure 5. The design originates from the scheme of the double-grating XUV ultrafast monochromator realized to select a suitable harmonic within the broad HHs spectrum without altering the intrinsic femtosecond pulse time duration [58]. The OPM is adopted since it gives higher efficiency in a compact environment.

The layout consists of four grazing-incidence parabolic mirrors, indicated as $\mathrm{P} 1, \mathrm{P} 2, \mathrm{P} 3$, and $\mathrm{P} 4$, and two plane gratings operated in the OPM, indicated as G1 and G2. The compressor is divided into two equal sections, P1-G1-P2 and P3-G2-P4. Since the gratings have to be operated in parallel light, the first mirror of each of the two sections acts as the collimator and the second mirror as the condenser. The four mirrors are operated with unity magnification; that is, the input arms $p$ of $\mathrm{P} 1$ and $\mathrm{P} 3$ are equal to the output arms $p$ of $\mathrm{P} 2$ and P4. The gratings G1 and G2 have the same groove density and altitude.

The use of parabolic mirrors instead of the more simple toroidal ones adopted for the design of femtosecond monochromators (see [43]) is required to reduce the aberrations to negligible levels, since any residual aberration causes a spread of the optical paths within the aperture of the instrument. The ideal collimator and condenser, completely free from aberrations in case of a point-like source, is the paraboloid. It can be shown that grazing-incidence toroidal surfaces when used as collimating and condensing elements exhibit residual aberrations that cannot be tolerated in the attosecond time scale (see [58]).

In brief, the input beam is collimated by $\mathrm{P} 1$ and then is diffracted by G1, that is, placed at a distance S2 from P2. The latter realizes a spectrally dispersed image of the source on its focal plane, that is, the source plane for the second section. The beam is collimated by P3 and diffracted by G2, that is, placed at a distance S3 from P3. Due to the symmetry of the double-grating configuration, all the rays diffracted from G2 are parallel and are finally focused on the output focal point by $\mathrm{P} 4$. The two focusing mirrors placed between the gratings act as a telescopic arrangement reducing the effective path between the gratings. This makes it possible to (1) produce an effectively negative grating separation with positive GDD, differently from the compressor with plane gratings; (2) continuously tune the GDD from negative to positive values; (3) achieve the very small grating separations necessary for the compensation of the attosecond chirp.

Since all the rays are collimated in the paths S1 between P1 and G1 and S4 between G2 and P4, the GD does not depend on the choice of S1 and S4 but only on the choice of S2 and $\mathrm{S} 3$, in particular on the sum $\mathrm{S}=\mathrm{S} 2+\mathrm{S} 3$. This is the key 


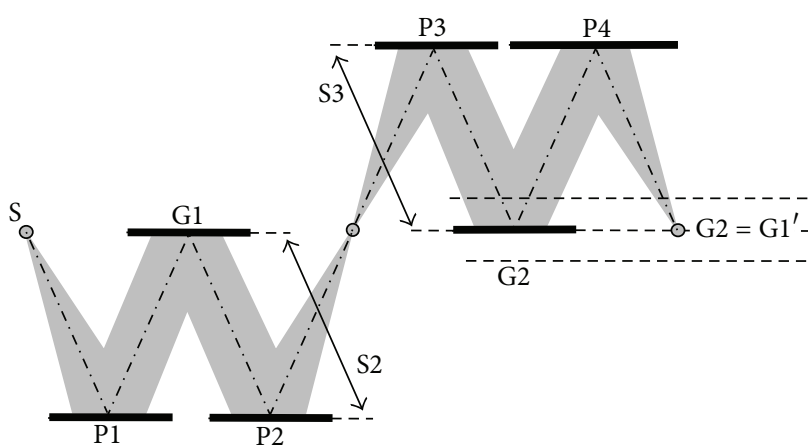

(a)

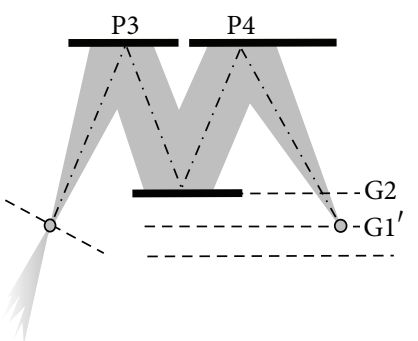

(b)

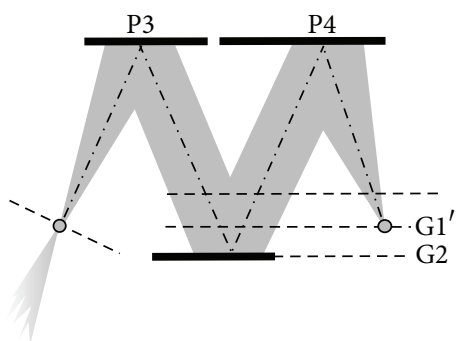

(c)

FIGURE 6: Operation of the configuration with variable GDD: (a) GDD =0; (b) GDD > 0; (c) GDD < 0 .

TABLE 2: Requirements for the attosecond compressor.

\begin{tabular}{lc}
\hline Bandwidth & $70-140 \mathrm{eV}(18-9 \mathrm{~nm})$ \\
Time stretching, $\Delta t$ & $0.47 \mathrm{fs}$ \\
Group delay dispersion, GDD & $-4.5 \cdot 10^{-3} \mathrm{fs}^{2}$ \\
\hline
\end{tabular}

TABLE 3: Parameters of the compressor for the $70-140 \mathrm{eV}$ spectral region.

\begin{tabular}{lc}
\hline Mirrors arms & $200 \mathrm{~mm}$ \\
Grating groove density & $200 \mathrm{gr} / \mathrm{mm}$ \\
Grating altitude & $1^{\circ}$ \\
Grating azimuth & $4.8^{\circ}$ \\
Distances S1, S2, and S4 & $50 \mathrm{~mm}$ \\
S3 & $378 \mathrm{~mm}$ \\
Total size of the compressor & $\approx 1.4 \mathrm{~m}$ \\
\hline
\end{tabular}

parameter to be properly set to introduce the required GDD. Due to the symmetry of the configuration, G1 is imaged on $\mathrm{G} 2$ when $\mathrm{S}=2 p$. This is the condition that gives group delay constant with the wavelength; that is, GDD $=0$. For $S<2 p$, G1 is imaged behind G2 and the resulting GDD is positive. For $\mathrm{S}>2 p, \mathrm{G} 1$ is imaged before G2 and the resulting GDD is negative, as shown in Figure 6. Therefore, the quantity $\mathrm{S}-2 p$ plays the role of $q$ in the double-grating compressor, being capable to give also negative values for $q$. The tuning of the GGD is performed in a simple way by moving G2 and keeping G1 fixed. Therefore, the compressor has fixed input and output points, fixed output direction, and a single mechanical movement to finely tune the GDD.

As a test case, the design of a compressor for attosecond pulses in the $70-140 \mathrm{eV}$ region is here discussed. Here, we assume the parameters of [59], which are reported in Table 2. The parameters of the compressor are reported in Table 3. The gratings have $200 \mathrm{gr} / \mathrm{mm}$ groove density and are operated at $1^{\circ}$ altitude and $4.8^{\circ}$ azimuth. The choice of the altitude has been done through ray-tracing calculations in order to minimize the spread of the optical paths of the rays with the same wavelengths, which states the ultimate limit of the time response at the output of the compressor, that results 12 as. The GD is shown in Figure 7. In such a large bandwidth,

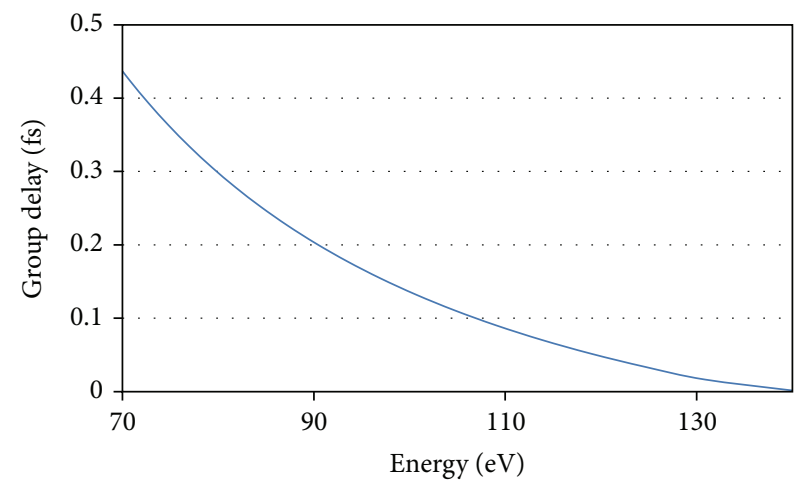

FIGURE 7: Group delay introduced by the XUV attosecond compressor with parameters of Table 3.

the GD is not linear, but it has also a quadratic term that originates third-order dispersion (TOD). It has been shown by simulation [59] that such a compressor is able to decrease the pulse duration down to $\approx 60$ as FWHM, which is not far from the Fourier limit on the given bandwidth (35 as).

Note that the double-grating configuration presented in the previous paragraph would give the same GDD for a G1G2 distance of $28 \mathrm{~mm}$, which is not practically feasible.

\section{Conclusions}

The use of grazing-incidence gratings to realize XUV compressors has been discussed for applications to HHs and FELs. Two different grating geometries, OPM and CDM, have been compared to show the peculiarities of both of them. The use of compressors may decrease the time duration of the pulse close to the Fourier limit and increase its peak intensity to offer full exploitation of femtosecond and subfemtosecond regime for ultrafast spectroscopy.

\section{Conflict of Interests}

The authors declare that there is no conflict of interests regarding the publication of this paper. 


\section{References}

[1] J. C. Diels and W. Rudolph, Ultrashort Laser Pulse Phenomena: Fundamentals, Techniques, and Applications on a Femtosecond Time Scale, Elsevier, New York, NY, USA, 2006.

[2] J. Marciak-Kozlowska and M. Kozlowski, From Femto-to Attoscience and Beyond, Nova Science Publishers, 2009.

[3] M. J. J. Vrakking and T. Elsaesser, "X-Ray photonics: X-rays inspire electron movies," Nature Photonics, vol. 6, no. 10, pp. 645-647, 2012.

[4] F. Lépine, M. Y. Ivanov, and M. J. J. Vrakking, "Attosecond molecular dynamics: fact or fiction?" Nature Photonics, vol. 8, no. 3, pp. 195-204, 2014.

[5] Z. Chang, A. Rundquist, H. Wang, M. M. Murnane, and H. C. Kapteyn, "Generation of coherent soft X Rays at $2.7 \mathrm{~nm}$ using high harmonics," Physical Review Letters, vol. 79, no. 16, pp. 2967-2970, 1997.

[6] M. Schnürer, Ch. Spielmann, P. Wobrauschek et al., "Coherent 0.5-keV X-ray emission from helium driven by a sub-10-fs laser," Physical Review Letters, vol. 80, no. 15, pp. 3236-3239, 1998.

[7] E. J. Takahashi, T. Kanai, K. L. Ishikawa, Y. Nabekawa, and K. Midorikawa, "Coherent water window $\mathrm{X}$ ray by phase-matched high-order harmonic generation in neutral media," Physical Review Letters, vol. 101, no. 25, Article ID 253901, 2008.

[8] P. B. Corkum and F. Krausz, "Attosecond science," Nature Physics, vol. 3, no. 6, pp. 381-387, 2007.

[9] F. Krausz and M. Ivanov, "Attosecond physics," Reviews of Modern Physics, vol. 81, no. 1, pp. 163-234, 2009.

[10] G. Sansone, L. Poletto, and M. Nisoli, "High-energy attosecond light sources," Nature Photonics, vol. 5, no. 11, pp. 655-663, 2011.

[11] F. Krausz and M. I. Stockman, "Attosecond metrology: from electron capture to future signal processing," Nature Photonics, vol. 8, no. 3, pp. 205-213, 2014.

[12] P. M. Paul, E. S. Toma, P. Breger et al., "Observation of a train of attosecond pulses from high harmonic generation," Science, vol. 292, no. 5522, pp. 1689-1692, 2001.

[13] R. López-Martens, K. Varjú, P. Johnsson et al., "Amplitude and phase control of attosecond light pulses," Physical Review Letters, vol. 94, no. 3, Article ID 033001, 2005.

[14] R. Kienberger, E. Goulielmakis, M. Uiberacker et al., "Atomic transient recorder," Nature, vol. 427, no. 6977, pp. 817-821, 2004.

[15] G. Sansone, E. Benedetti, F. Calegari et al., "Isolated singlecycle attosecond pulses," Science, vol. 314, no. 5798, pp. 443-446, 2006.

[16] M. Schultze, E. Goulielmakis, M. Uiberacker et al., "Powerful 170-attosecond XUV pulses generated with few-cycle laser pulses and broadband multilayer optics," New Journal of Physics, vol. 9, article 243, 2007.

[17] K. Zhao, Q. Zhang, M. Chini, Y. Wu, X. Wang, and Z. Chang, "Tailoring a 67 attosecond pulse through advantageous phasemismatch," Optics Letters, vol. 37, no. 18, pp. 3891-3893, 2012.

[18] M. Chini, K. Zhao, and Z. Chang, "The generation, characterization and applications of broadband isolated attosecond pulses," Nature Photonics, vol. 8, no. 3, pp. 178-186, 2014.

[19] A. Fleischer, O. Kfir, T. Diskin, P. Sidorenko, and O. Cohen, "Spin angular momentum and tunable polarization in highharmonic generation," Nature Photonics, vol. 8, pp. 543-549, 2014.

[20] F. Mauger, A. D. Bandrauk, A. Kamor, T. Uzer, and C. Chandre, "Quantum-classical correspondence in circularly polarized high harmonic generation," Journal of Physics B: Atomic, Molecular and Optical Physics, vol. 47, no. 4, Article ID 041001, 2014.
[21] Y. Mairesse, A. De Bohan, L. J. Frasinski et al., "High-harmonics chirp and optimization of attosecond pulse trains," Laser Physics, vol. 15, no. 6, pp. 863-870, 2005.

[22] "Free-electron laser FLASH," http://flash.desy.de/.

[23] SACLA (XFEL), November 2014, http://xfel.riken.jp/eng/.

[24] "LCLS-Linac Coherent Light Source," http://lcls.slac.stanford $. e d u /$.

[25] FERMI, 2014, http://www.elettra.trieste.it/FERMI/.

[26] P. J. Emma, K. Bane, M. Cornacchia et al., "Femtosecond and subfemtosecond X-ray pulses from a self-amplified spontaneous-emission-based free-electron laser," Physical Review Letters, vol. 92, no. 7, Article ID 074801, 2004.

[27] Y. Ding, Z. Huang, D. Ratner, P. Bucksbaum, and H. Merdji, "Generation of attosecond x-ray pulses with a multicycle twocolor enhanced self-amplified spontaneous emission scheme," Physical Review Special Topics-Accelerators and Beams, vol. 12, no. 6, Article ID 060703, 2009.

[28] T. Tanaka, "Proposal for a pulse-compression scheme in X-ray free-electron lasers to generate a multiterawatt, attosecond $\mathrm{X}$ ray pulse," Physical Review Letters, vol. 110, no. 8, Article ID 084801, 2013.

[29] J. B. Rosenzweig, D. Alesini, G. Andonian et al., "Generation of ultra-short, high brightness electron beams for single-spike SASE FEL operation," Nuclear Instruments and Methods in Physics Research, Section A: Accelerators, Spectrometers, Detectors and Associated Equipment, vol. 593, no. 1-2, pp. 39-44, 2008.

[30] Y. Ding, F.-J. Decker, P. Emma et al., "Femtosecond X-ray pulse characterization in free-electron lasers using a cross-correlation technique," Physical Review Letters, vol. 109, no. 25, Article ID 254802, 2012.

[31] C. Pellegrini, "High power femtosecond pulses from an Xray SASE-FEL," Nuclear Instruments and Methods in Physics Research, Section A: Accelerators, Spectrometers, Detectors and Associated Equipment, vol. 445, no. 1-3, pp. 124-127, 2000.

[32] X. Shu, T. Peng, and Y. Dou, "Chirped pulse amplification in a free-electron laser amplifier," Journal of Electron Spectroscopy and Related Phenomena, vol. 184, no. 3-6, pp. 350-353, 2011.

[33] C. Feng, L. Shen, M. Zhang, D. Wang, Z. Zhao, and D. Xiang, "Chirped pulse amplification in a seeded free-electron laser for generating high-power ultra-short radiation," Nuclear Instruments and Methods in Physics Research, Section A: Accelerators, Spectrometers, Detectors and Associated Equipment, vol. 712, pp. 113-119, 2013.

[34] L. Poletto, G. Tondello, and P. Villoresi, "High-order laser harmonics detection in the EUV and soft X-ray spectral regions," Review of Scientific Instruments, vol. 72, no. 7, pp. 2868-2874, 2001.

[35] L. Poletto, S. Bonora, M. Pascolini, and P. Villoresi, "Instrumentation for analysis and utilization of extreme-ultraviolet and soft $\mathrm{x}$-ray high-order harmonics," Review of Scientific Instruments, vol. 75, no. 11, pp. 4413-4418, 2004.

[36] L. Poletto and F. Frassetto, "Time-preserving grating monochromators for ultrafast extreme-ultraviolet pulses," Applied Optics, vol. 49, no. 28, pp. 5465-5473, 2010.

[37] P. Villoresi, "Compensation of optical path lengths in extremeultraviolet and soft-X-ray monochromators for ultrafast pulses," Applied Optics, vol. 38, no. 28, pp. 6040-6049, 1999.

[38] L. Poletto, “Time-compensated grazing-incidence monochromator for extreme-ultraviolet and soft X-ray high-order harmonics," Applied Physics B: Lasers and Optics, vol. 78, no. 7-8, pp. 1013-1016, 2004. 
[39] F. Frassetto, C. Cacho, C. A. Froud et al., "Single-grating monochromator for extreme-ultraviolet ultrashort pulses," Optics Express, vol. 19, no. 20, pp. 19169-19181, 2011.

[40] C. Grazioli, C. Callegari, A. Ciavardini et al., "CITIUS: an infrared-extreme ultraviolet light source for fundamental and applied ultrafast science," Review of Scientific Instruments, vol. 85, no. 2, Article ID 023104, 2014.

[41] L. Poletto, P. Miotti, F. Frassetto et al., "Double-configuration grating monochromator for extreme-ultraviolet ultrafast pulses," Applied Optics, vol. 53, pp. 5879-5888, 2014.

[42] P. Heimann, O. Krupin, W. F. Schlotter et al., "Linac coherent light source soft X-ray materials science instrument optical design and monochromator commissioning," Review of Scientific Instruments, vol. 82, no. 9, Article ID 093105, 2011.

[43] L. Poletto and P. Villoresi, "Time-delay compensated monochromator in the off-plane mount for extreme-ultraviolet ultrashort pulses," Applied Optics, vol. 45, no. 34, pp. 8577-8585, 2006.

[44] L. Poletto, P. Villoresi, E. Benedetti et al., "Intense femtosecond extreme ultraviolet pulses by using a time-delay-compensated monochromator," Optics Letters, vol. 32, no. 19, pp. 2897-2899, 2007.

[45] L. Poletto, P. Villoresi, F. Frassetto et al., "Time-delay compensated monochromator for the spectral selection of extremeultraviolet high-order laser harmonics," Review of Scientific Instruments, vol. 80, no. 12, Article ID 123109, 2009.

[46] M. Ito, Y. Kataoka, T. Okamoto, M. Yamashita, and T. Sekikawa, "Spatiotemporal characterization of single-order high harmonic pulses from time-compensated toroidal-grating monochromator," Optics Express, vol. 18, no. 6, pp. 6071-6078, 2010.

[47] H. Igarashi, A. Makida, M. Ito, and T. Sekikawa, "Pulse compression of phase-matched high harmonic pulses from a timedelay compensated monochromator," Optics Express, vol. 20, no. 4, pp. 3725-3732, 2012.

[48] L. Poletto, P. Azzolin, and G. Tondello, "Beam-splitting and recombining of free-electron-laser extreme-ultraviolet radiation," Applied Physics B: Lasers and Optics, vol. 78, no. 7-8, pp. 1009-1011, 2004.

[49] F. Frassetto, P. Villores, and L. Poletto, "Beam separator for high-order harmonic radiation in the 3-10 nm spectral region," Journal of the Optical Society of America A, vol. 25, no. 5, pp. 1104-1114, 2008.

[50] L. Poletto, F. Frassetto, and P. Villoresi, "Ultrafast grating instruments in the extreme ultraviolet," IEEE Journal on Selected Topics in Quantum Electronics, vol. 18, no. 1, pp. 467-478, 2012.

[51] L. Poletto and F. Frassetto, "Design of high-resolution grazingincidence echelle monochromators," Applied Optics, vol. 48, no. 28, pp. 5363-5370, 2009.

[52] M. Nevière, "Experimental verifications and applications of the theory," in Electromagnetic Theory of Gratings, R. Petit, Ed., chapter 4, Springer, Berlin, Germany, 1980.

[53] M. Pascolini, S. Bonora, A. Giglia, N. Mahne, S. Nannarone, and L. Poletto, "Gratings in a conical diffraction mounting for an extreme-ultraviolet time-delay-compensated monochromator," Applied Optics, vol. 45, no. 14, pp. 3253-3262, 2006.

[54] O. E. Martinez, "3000 times grating compressor with positive group velocity dispersion: application to fiber compensation in 1.3-1.6 $\mu \mathrm{m}$ region," IEEE Journal of Quantum Electronics, vol. 23, no. 1, pp. 59-64, 1987.

[55] O. E. Martinez, "Design of high-power ultrashort pulse amplifiers by expansion and recompression," IEEE Journal of Quantum Electronics, vol. 23, no. 8, pp. 1385-1387, 1987.
[56] L. Walmsley, L. Waxer, and C. Dorrer, "The role of dispersion in ultrafast optics," Review of Scientific Instruments, vol. 72, no. 1, pp. 1-29, 2001.

[57] F. Frassetto, L. Giannessi, and L. Poletto, "Compression of XUV FEL pulses in the few-femtosecond regime," Nuclear Instruments and Methods in Physics Research Section A, vol. 593, no. 1-2, pp. 14-16, 2008.

[58] F. Frassetto, P. Villoresi, and L. Poletto, "Optical concept of a compressor for XUV pulses in the attosecond domain," Optics Express, vol. 16, no. 9, pp. 6652-6667, 2008.

[59] M. Mero, F. Frassetto, P. Villoresi, L. Poletto, and K. Varjú, "Compression methods for XUV attosecond pulses," Optics Express, vol. 19, no. 23, pp. 23420-23428, 2011. 

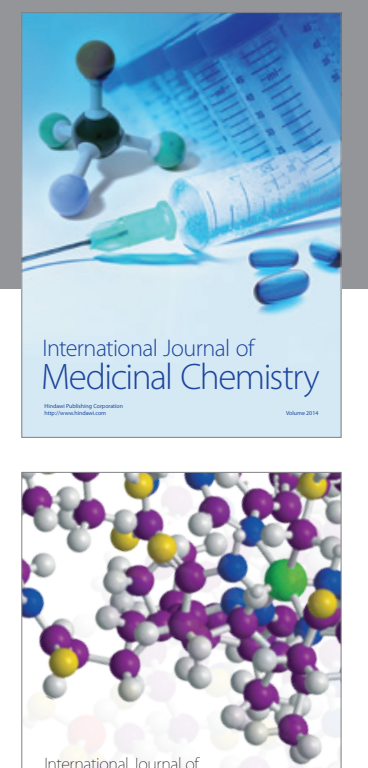

\section{Carbohydrate} Chemistry

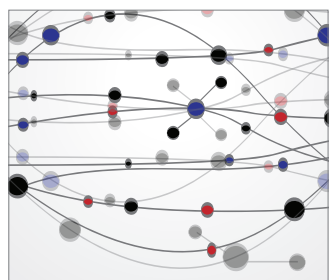

The Scientific World Journal
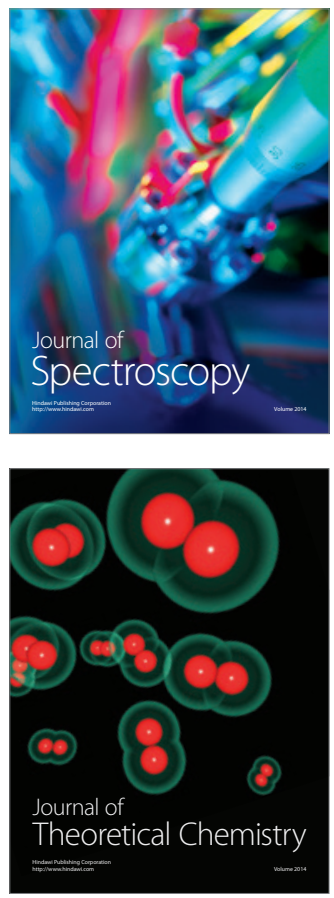
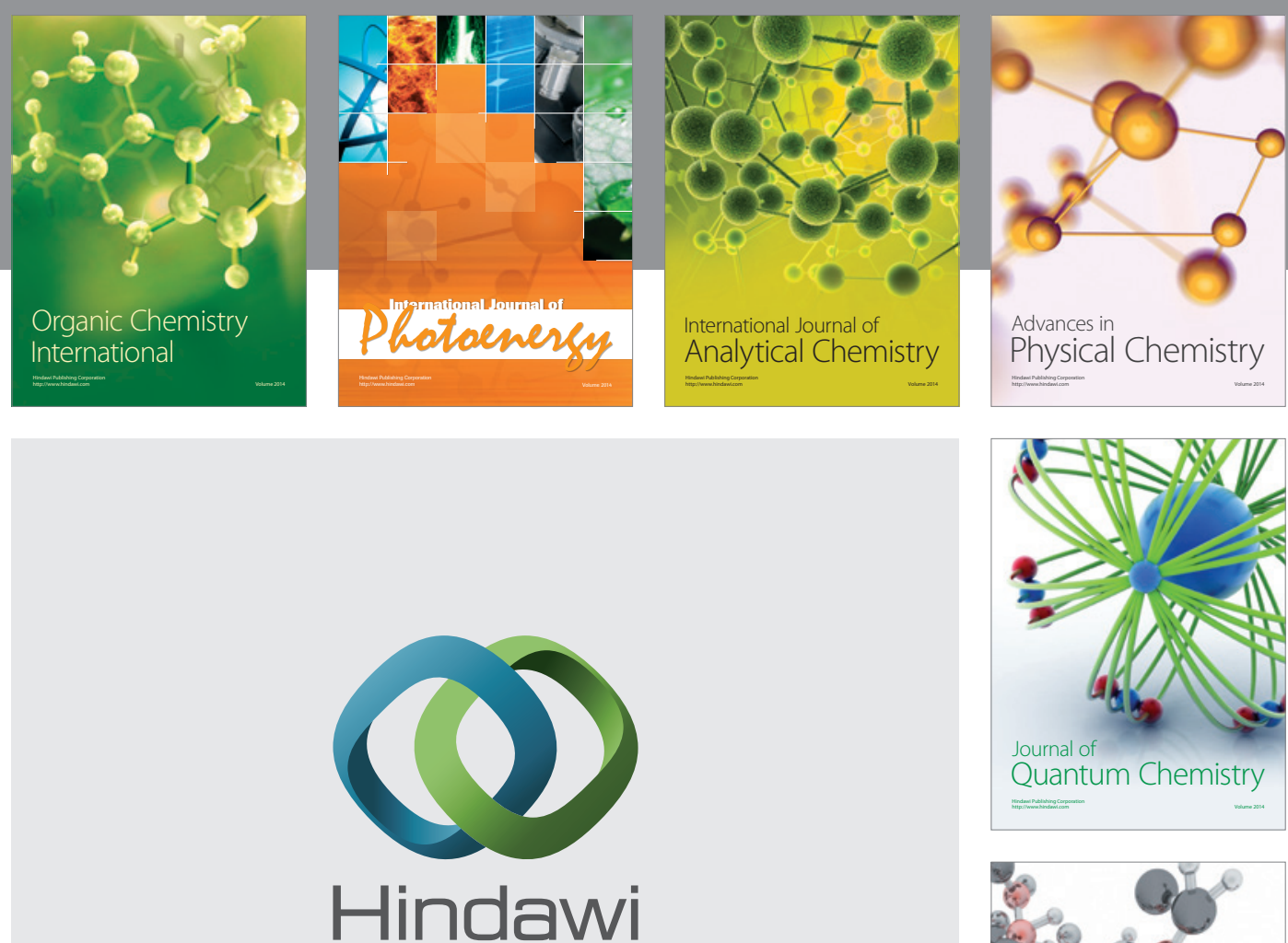

Submit your manuscripts at

http://www.hindawi.com

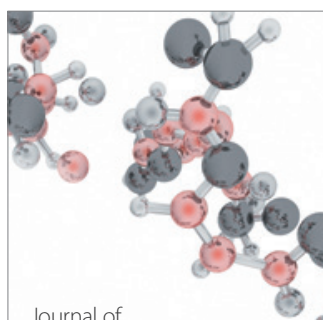

Analytical Methods

in Chemistry

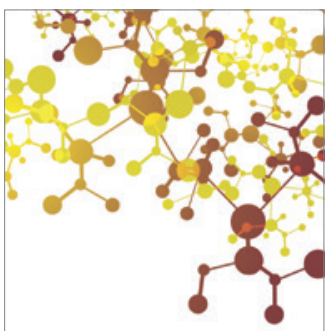

Journal of

Applied Chemistry

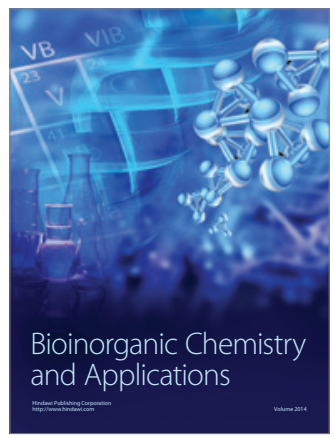

Inorganic Chemistry
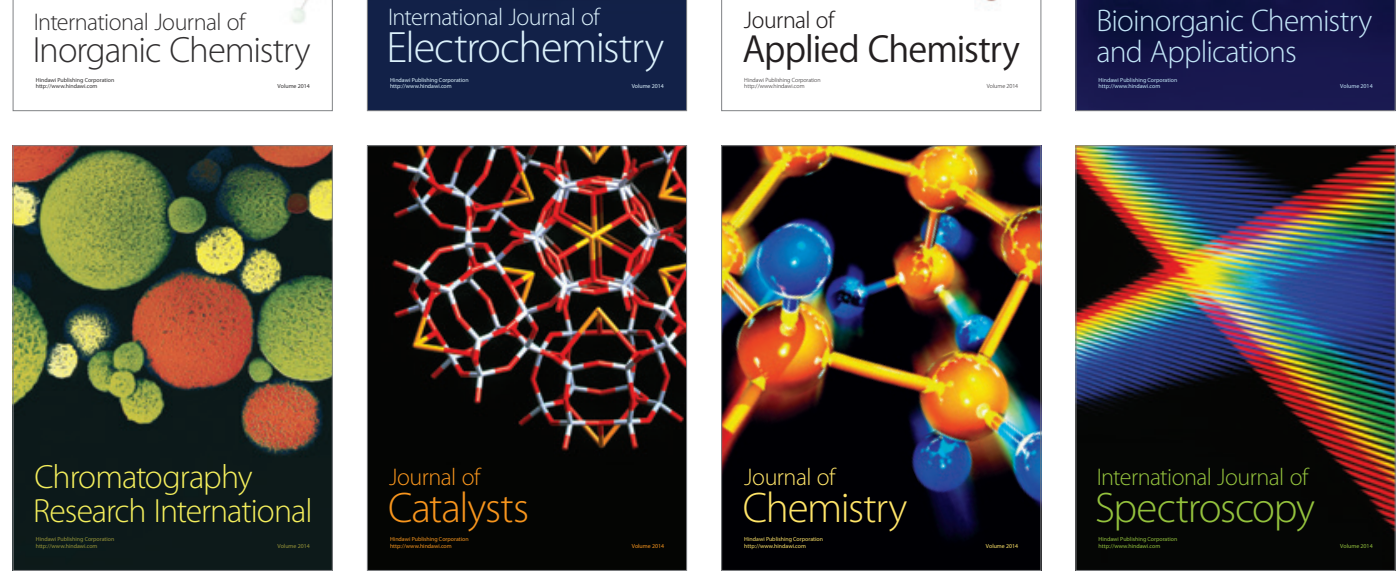